\title{
PARACONSISTENCY AND ITS PHILOSOPHICAL INTERPRETATIONS
}

\author{
EDUARDO BARRIO AND BRUNO DA RÉ
}

\begin{abstract}
Many authors have considered that the notions of paraconsistency and dialetheism are intrinsically connected, in many cases, to the extent of confusing both phenomena. However, paraconsistency is a formal feature of some logics that consists in invalidating the rule of explosion, whereas dialetheism is a semantical/ontological position consisting in accepting true contradictions. In this paper, we will argue against this connection and show that it is perfectly possible to adopt a paraconsistent logic and reject dialetheism, and, moreover, that there are examples of non-paraconsistent logics that can be interpreted in a dialetheic way.

Keywords: Paraconsistency - Dialetheia - Philosophical Interpretation
\end{abstract}

\section{INTRODUCTION}

Logics are often accompanied by philosophical interpretations. For example, several systems of modal logics talk about possible worlds and relations of accessibility, intuitionistic logic is connected with constructive proofs, and relevance logics are linked with situations. In this regard, the role of philosophical interpretations has been highlighted by Richard Routley. For instance, he has affirmed that "a satisfactory sentential logic should include however, especially if philosophical applications are intended, a relevant implication" [28, p.305], justifying the adoption of a formal system in virtue of some particular philosophical interpretation. In the more particular context of paraconsistent logics, the discussion about philosophical interpretations has centered on whether there are dialetheia or whether there is simply contradictory evidence. Concerning the first case, many authors have considered that the notions of paraconsistency and dialetheism are intrinsically connected, in many cases, to the extent of confusing both phenomena. However, on the one hand, paraconsistency is a formal feature of some logics that consists in abandoning at least the rule of explosion. On the other hand, dialetheism is a semantical/ontological position consisting in accepting true contradictions. In this paper, we want to focus on an issue that has not yet been sufficiently explored: how a logical theory is connected to its philosophical interpretations. In particular, we will argue against the existence of a close relationship between both elements. In other words, we would like to conclude that there is not any single canonical philosophical interpretation for logical systems. We will display that it is perfectly possible to adopt a paraconsistent logic and reject dialetheism. Also, we will provide examples of non-paraconsistent logics that can be interpreted in a dialetheic way. In sum, we will show that there is no any necessary connection between paraconsistency and one philosophical interpretation. In order to do so, firstly, in Section 2 we are going to explain the main ideas developed around the notion of dialetheism. Next, in Section 3 we

Australasian Journal of Logic (15:2) 2018, Article no. 3.1 
will summarize the different definitions given in the literature of paraconsistency. In Section 4 we will present the distinction between pure and apply logics, and the relevance for our main purposes. we are going to present the distinction between pure and apply logics, and the relevance for our main purposes. In Section 5, we will argue that it is perfectly possible to adopt a paraconsistent logic and to be non dialetheist. In Section 6, we will show that it is possible to embrace a dialetheist position without adopting any paraconsistent logic. Moreover, we will present some examples of non-paraconsistent logics that are explicitly committed with inconsistent objects. Therefore, we will distinguish between paraconsistency and inconsistency. Finally, we will conclude with some closing remarks.

\section{Dialetheism}

Dialetheism is the view that there are true contradictions. Many philosophers have adopted this thesis. For instance, this perspective was defended by Priest (e.g. [19], [22]) and also by Routley, (e.g. in [28] and [29]), among others. Usually, a sentence is called a dialetheia if, semantically speaking, is true and false, or expresses a true contradiction.

Two thesis are involved in the dialetheism:

Definition 2.1. Dialetheism is the thesis that some contradictions are true.

Definition 2.2. Dialetheism is the thesis that some sentences are both true and false.

There are several motivations to endorse a dialetheist view, among them: semantic and other paradoxes linked with self-reference, many of our beliefs, legal codes, Meinongian objects, Hegelian and dialectic philosophy, rationality, and scientific theories such quantum mechanics, among others.

As we have seen, the formulation of the dialetheist thesis involves the concept of truth. But it is well known that there are different notions of truth and that, therefore, there are different ways of adhering to the dialetheism. What is at stake is whether dialetheism brings with it certain ontological commitments. True contradictions could imply a commitment to the existence of inconsistent entities as their ontological counterparts. For example, if there were good reasons to adopt the correspondence view about truth, then there would also be good reasons to connect the dialetheism with the existence of entities of a specific nature. Then, when $\mathrm{A}$ is a dialetheia, there would have to be entities in virtue of $\mathrm{A}$ and $\neg A$ are both true. Berto [7] analyzes this option in detail. And leaving aside the question about the correspondence theory of truth, Priest offers good reasons to accept inconsistent entities in the natural and social world ([22, Ch. 12 and 13]) However, some sentences could be both true and false, but this result does not imply any ontological commitment. Such an option seems to be available if a deflationary position about truth were adopted. In this line, the sympathy with the dialetheism is compatible with the rejection of the existence of inconsistent entities. Or at least, with neutrality about this question. Beall seems to adopt this position in [6]. In both cases, that is, if it remains only at the semantic level or if the additional step is taken to the ontological level, this decision will not be a consequence of supporting the dialetheism. As Priest affirms [22], this point of view is not necessarily committed to any particular conception of the notion of truth.

Australasian Journal of Logic (15:2) 2018 Article no. 3.1 
Dialetheism, the view that some contradictions are true, does not commit one per se to any particular account of truth. A dialetheist who holds a correspondence account of truth will hold that there are inconsistent facts (whatever, in the end, this is taken to mean). One who holds a verificationist/pragmatist account of truth will hold that some contradictions are warranted by the evidence, and so on.

Notwithstanding this, the defense of a particular approach to truth seems to bring with it different ways of adopting the view according to which there are true contradictions.

In addition, beyond the ontological and semantic aspects, there is another important distinction that involves the scope of the dialetheist thesis.

Definition 2.3. Trivialism is the view according to all contradictions are true.

It is clear that trivialism implies dialetheism. But at least it is not obvious that the other way around works. A dialetheist typically only holds that some (and, usually, some specific cases as paradoxes) sentences are dialetheia. Nevertheless, it is not mandatory to this view to support that all sentences are true and false. Distinguishing normal cases of special cases in which a sentence can be true and false seems to be one of the proposals to be achieved.

In short, dialetheism is a family of semantic/ontological approaches. There are many aspects both ontological and semantic that can change from one position to another. Options within the real or the possible or various ways in which we might understand the truth of a contradiction determine a variety of approaches that possess critical philosophical disagreements $^{1}$.

\section{PARACONSISTENCY}

As well as the case of dialetheism, there are also several motivations to adopt a paraconsistent logic. One of them is to deal with inconsistent information. The idea is not to reject theories or sets of sentences in virtue of their inconsistency, but to deal with them employing a logic that is tolerant to contradictions. As we will formally pose below, a theory is explosive if, from inconsistent premises, it is possible to entail everything. Paraconsistent logics are not explosive.

Another possible motivation is dialetheism: true contradictions should not cause triviality. IIn both cases, the key is that paraconsistent logics allow for the capturing the capability of reasoning with inconsistent premises without entailing everything. Since dialetheism is committed to accepting true contradictions, it seems that a paraconsistent notion of logical consequence allows them to prevent triviality. By contrast, prima facie, an explosive notion of logical consequence is not a suitable instrument to treat inconsistent information ${ }^{2}$. For instance, one of the definitions of paraconsistency is the following, from [32]: "A logic $\mathcal{L}$ is paraconsistent if it can support inconsistent non-trivial theories".

\footnotetext{
${ }^{1}$ For some further discussion of these issues, see [23].

${ }^{2}$ This thesis, as plausible as it sounds, we think is not true. In Section 6 we will discuss it in detail.
} 
More formally, a logic $\mathcal{L}$ is paraconsistent if the inference of explosion is invalid in it. This means that

$$
\text { (Explosion) } A, \neg A \vDash_{\mathcal{L}} B^{3}
$$

is not valid.

It is important to note that there are at least two notions of paraconsistency involved in this definition. It is important to note that there are at least two notions of paraconsistency involved in this definition. Following Ripley [27], one could refer to the following as conjunctive paraconsistency and collective paraconsistency:

Definition 3.1. (Conjunctive paraconsistency): A consequence relation $\vdash$ on a language $L$ is conjunctively paraconsistent iff there are wffs $A, B \in L$ such that $A \wedge \neg A \nvdash B$

Definition 3.2. (Collective paraconsistency): A consequence relation $\vdash$ on a language $L$ is conjunctively paraconsistent iff there are wffs $A, B \in L$ such that $A, \neg A \nvdash B$.

Only in a logic for which $A \wedge B \vdash C$ implies $A, B \vdash C$ does collection-paraconsistency imply conjunction-paraconsistency (see Ripley [27, p.772]).

However, beyond these options, paraconsistent logics are closely related to other kinds of non-classical logics [U+FFFD] specially to relevant logics and many-valued logics. Let [U+FFFD] note from the above definitions that paraconsistency is a property not only of the notion of logical consequence, but also of negation. In other words, the presence of some kind of negation is necessary for this definition.

Nevertheless, as Omori argues in [12, p.825] "the understanding of the only connective involved in the explosion, namely negation, is not shared among paraconsistentists". However, what is clear is that the negation involved in any paraconsistent system is not classical (in the sense of truth and falsity are exhaustive and exclusive). But it is not completely clear how to identify only one positive condition: maybe paraconsistent negations are not exclusive ${ }^{4}$.

However, all of these definitions of paraconsistency are negative. As Urbas [32, p.344] notes:

it serves negatively to disqualify explosive logics as clearly inadequate for reasoning in inconsistent situations, but it does nothing positively to illuminate those that remain. All that it tells us about paraconsistent deducibility is that a certain inference is rejected, leaving it otherwise entirely open which inferences are to be accepted.

\footnotetext{
${ }^{3}$ Here, we are using $\vDash$, but depending on the context, we can speak in terms of syntactic consequence. Actually, in this section we will use $\vdash$ and in Section 6 we will use this and other definitions replacing $\vDash$ or $\vdash$ with $\Rightarrow$.

${ }^{4}$ The most radical point of view on this point has been adopted by Slater. In [31] Slater analyzes the notion of contradictoriness to try to show that negation in paraconsistent logics is merely a subcontrary forming operator and not one which forms contradictories. There are many answers to this argument. For instance, see Oller in [16] or Restall in [24], among others.
}

Australasian Journal of Logic (15:2) 2018 Article no. 3.1 
Notably, da Costa, in his pioneering work on C-Systems, [11, p.498], suggested that all paraconsistent logics must meet four conditions:

- The principle of contradiction $\neg(A \wedge \neg A)$ must not be a theorem.

- From two contradictory formulae it must be impossible to deduce an arbitrary formula.

- The logic must have a simple first-order extension.

- The logic must contain as much of classical logic that does not impede the fulfillment of conditions 1-3) as possible.

Condition 2 is the rejection of explosion. But the other conditions add some information (not only negative) on what a paraconsistent logic could be.

But even if it is not so important to give a positive definition of what is a paraconsistent logic, there are other problems about these definitions in term of failure of some kind of explosion. Some scholars - such as Urbas or Fjellstad - consider that this strategy to characterize a paraconsistent logic is that it is neither very restrictive nor substantive.

For example, it is important to note that there are several logics such as Johansson's 'Minimal Calculus' [13], a positive fragment of intuitionistic logic, in which although explosion is not a valid rule, a special instance of explosion such as $A, \neg A \vDash_{\mathcal{L}} \neg B$ is. Other cases are some da Costa's logics that can avoid the general formulation of explosion but deliver $A, \neg A \vDash_{\mathcal{L}} B \rightarrow C$.

This is not the best news. We could want to exclude not only absolute explosiveness, but also explosiveness which is specific to particular connectives. Maybe, it could be a good idea to try to add conditions to exclude this type of logics. So, as Priest points out ([18, p.4]): "paraconsistency, in the way defined, is not a sufficient condition for a consequence relation to be a sensible one with which to handle inconsistent information". In this direction, Urbas [32, 345-354] analyses several options. In principle, one wants a definition of paraconsistency that ensures for any unary placed connective $*$ and any formulae $A, B$ and $C$, both $A, \neg A \nvdash * B$ and $A, \neg A \nvdash B * C$.

After all, the underlying philosophical motivation for paraconsistent logics is to avoid any kind of trivialities from inconsistent premise sets. Then, Urbas [32, p.346] propose that a theory is $*$-trivial if it contains all expressible sentences having as principal connective. And using this characterization, one has that a logic $\mathrm{L}$ is $*$-paraconsistent if it can support inconsistent $*$-non-trivial theories.

According to Urbas (1990 p.346), this condition ensures that $*$-connectives "do a better job of containing the deductive damage caused by inconsistency".

Obviously some cases with definable connectives like the equivalency, $B * C$ is $B \rightarrow C$ and $C \rightarrow B$ could determine cases of trivialities like $A, \neg A \nvdash B * C$. Other potential problems are cases as $A, \neg A \nvdash B \rightarrow B$.

All these examples could show that connection between explosion and triviality is more complicated that what usually is admitted. Some logics allow some particular cases of triviality from inconsistent premises that could be tolerated by some paraconsistent logics.

Australasian Journal of Logic (15:2) 2018 Article no. 3.1 
A further case that generates discussion is pointed out by Barrio, Pailos and Szmuc ([3]). According to them, the substructural logic TS that lacks valid inferences should be considered to be a paraconsistent logic. Against this point, Fjellstad considers that the conclusion that TS is paraconsistent shows that an important (conceptual) aspect of paraconsistency is missing from the definition connected with the rejection of explosion. According to him, paraconsistency is linked exclusively with allowing reasoning with inconsistent information. The rejection of explosion should be considered as a move to permit contradictions without trivializing the theory. But this would be the case regarding TS since TS is not inconsistent. Explosion fails in TS, but this would be a casualty of inconsistency.

But - as Barrio, Szmuc and Pailos reply - the fact that TS has no valid inferences does not allow one to identify its inferential consequence relation with the empty set. For, in a certain sense, the fact that TS has no valid inferences is dependent on the language employed. If we have a constant $T$ representing the value $t$, and a constant $\perp$ representing the value $f$, then e.g. the following inferences will be valid in this extension of TS (and, thus, of the 3-valued Kleene algebra):

$$
\top \Rightarrow \top, \perp \Rightarrow \perp \vDash_{\mathcal{L}} \perp \Rightarrow \top
$$

And, more importantly, the addition of such constants to TS will not imply the validity of explosion at the inferential level. Therefore, the authors claim, TS is a paraconsistent logic in a meaningful and non-trivial sense.

However, in this context, it does not matter if we hold that TS is the empty logic or not. The point seems to persist because we can focus on the logic based on the language without constants.

Another interesting case, suggested by Francesco Paoli, is to take paraconsistency as an abstract notion that should not presuppose the concept of negation. So, the question would be whether it is possible to give an abstract characterization of paraconsistency independently of the negation. In the metaleinferential level, one possible way to go is to define the metainferential notion of paraconsistency regarding invalidating the metainference:

$$
\Rightarrow A \quad A \Rightarrow
$$

So, following Paoli, this definition would also have the further advantage of allowing the formulation of a metainferential version of the dual notion of paracompleteness. However, it is not clear how - and probably is not possible - to apply this procedure to the basic case of the explosion at the inferential level. At this level, it does not seem possible to characterize the rule of explosion without negation.

We think that his variety of definitions is a consequence of the complexity of the problem of uniquely characterizing the set of paraconsistent logics. In Priest's words ([18, p.130]):

(...) it seems unlikely that there is any purely formal necessary and sufficient condition for the spirit of paraconsistency: inconsistent information may

Australasian Journal of Logic (15:2) 2018 Article no. 3.1 
make a nonsense of a consequence relation in so many, and quite different, ways.

The aim of this section was to show that the notion of paraconsistency is more complex than what is usually thought. And it would be really complicated to offer purely formal conditions for a paraconsistent logic. So, although giving a unique definition of paraconsistency, compatible with every account taken in the literature, seems an impossible task, we found that in every proposal, paraconsistency requires at least the existence of a counterexample to explosion. So, this seems to be the common ground of all of the accounts, and in this way, we will use this concept in this paper unless there is any clarification. In the following section, once we present the distinction between pure, applied logics and philosophical interpretation, we will give further arguments for considering explosion to be the distinctive feature of praconsistency.

\section{Pure and Applied Logics: their philosophical interpretations}

In this section, adopting the Priest's distinction between pure and applied logics, we are going to analyze what happens with the relation between paraconsistent logical systems (pure and applied) and their philosophical interpretations. Specifically, we are interested in rejecting the idea according to which dialetheism should be considered as the canonical interpretation of some paraconsistent logic. Let's firstly contrast between pure and applied logics.

On the one hand, one can be interested in pure systems of logic. In this sense, given a set of sentences, logic should show us what is follows from these sentences. A good argument is one whose premises entails its conclusion; its conclusion is a consequence of its premises. Logic should be very general, abstract and topic-neutral. One should pay no attention to the subject matter of the sentences: when one is interested in logical properties, it does not matter what sentences are talking about. It does not matter either what is known or the epistemic source of the reasons to support a premise. What is only important is to pay attention to the logical relationship between them. With the proposal of explaining this notion, one develops several approaches to give a theory of what is entailed from what. Then, proof-theoretical account tries to explain how to prove something from something. They can be presented by sequent calculus, natural deduction, axiomatic systems. These theories have been developed to focus on different aspect of proofs. Pure logics also can be presented by models. Model theoretical approach tries to explain how to preserve semantics designated values from semantics values. Set-theoretical models, plurals models, non-deterministic semantics, partial valuations, Kripke's models, etc. have been developed to illuminate the notion of validity. Models and proof are mathematical instruments to understand the concept of consequence. Generally speaking, soundness and completeness are properties that pure systems should have. In this sense, any evaluation of a pure logic is based on the fulfillment of those properties. The search for results of soundness and completeness is a central goal at this level. The deduction and the cut-elimination

Australasian Journal of Logic (15:2) 2018 Article no. 3.1 
theorems, the interpolation results, the compactness of models and the decidability of a system of proof are fundamentals elements to evaluate a pure logic ${ }^{5}$

In Priest's words:

First, there are numerous pure logics. This point I take to be relatively uncontentious. There are the many-valued logics that Lukasiewicz invented, not to mention others such as intuitionism, quantum logic, and paraconsistent logic (one of which, LP, we met in the preceding sections). Possibly, a purist might say that they are not logics since they are not the real logic. But that would be like saying that non-Euclidean geometries are not geometries since they are not the real geometry. In both cases we have a family of structures (logics or geometries) that are perfectly well-defined mathematical structures; and, as far as that goes, all on a par. [21, p. 164-165]

On the other hand, one can be interested in the theoretical applications of logic ${ }^{6}$. According to this proposal, logics can be applied to several domains. Their domains of applications range from computer science and artificial intelligence, electrical circuits to the grammar of natural languages. There are several logics that can be applied to a given domain although a given logic is usually more adequate to certain domains than to others.

For example, according to Bueno [9, p.542],

both classical logic and paraconsistent logic can be applied to consistent domains, and typically they will generate the same results there. However, once we move to inconsistent domains, classical logic is no longer an adequate option (unless we want to allow the arbitrary rejection of some bits of information about such domains). After all, by identifying inconsistency and triviality, classical logic precludes the possibility that, in inconsistent domains, we fail to validly infer at least one sentence of the language we use.

As regards applied logic, one could be interested in which of the infinitely many paraconsistent logics is the right one. In this regard, there are at least two options. First, one could support the idea that the answer depends on the kind of inconsistency which we are dealing with. Maybe more of only one logic might have a nice application. Second, one could claim that there is only one canonical application for a logic. This is Priest's view. The canonical application of logic is namely the reasoning in natural language. Then, according to Priest [20], only canonical pluralism provides an interesting case for logical pluralism. After all, pure logical pluralism and theoretical pluralism only claim that there are different logicsan undeniable, but ultimately uninteresting, mathematical fact.

\footnotetext{
${ }^{5}$ Richard Routley has linked this factor with the question about the adequacy of a logic. According to him, there are some internal systemic reasons (for instance, in [28, p.318] he says: "Among the questions of adequacy that arise for the dialectical theories outlined, a most important initial one is: Are the theories non-trivial?").

${ }^{6}$ Richard Routley has also accepted that there are some external reasons that allow for evaluating the adequacy of a logic: for example, [28, p.305],"if a logic practically can fulfill the most important business of furnishing a logical basis for naïve dialectical set theory and for semantically closed natural languages".
}

Australasian Journal of Logic (15:2) 2018 Article no. 3.1 
Nevertheless, according to Priest and as opposed to Bueno[U+FFFD] view, as applied logics, classical and paraconsistent logics cannot both be right. As is well known, classical logics and paraconsistent logics seem to disagree with the notion of negation. In this regard, the main question is: What kind of restrictions does negation put on any theory about negation? From Priest's view, when a system of logic is going to be understood as applied logic, then a theory about negation has a specific subject matter. Negation only can be a theory about a contradictory forming operator. So, any account of negation that fails in delivering a contradictory forming operator is not eligible as a true account of negation. And concerning logical pluralism at the level of applied logics, this means that the restrictions on negation allow that only one logic could be applied correctly to the inferences we make in natural language.

To summarize the discussion: there are pure and applied logics. There are infinitely many pure logics. There are a lot of different applications. But maybe, if Priest is right, only one is the canonical (the human reasoning) and only one pure system can be applied to this domain.

In any case, the main question is whether there is the possibility of imposing enough formal constraints on a pure logic in such a way that when we apply it to the inferences we make in language, a single philosophical interpretation is determined.

It is clear that one can use philosophical interpretations to try to obtain additional understanding of certain pure logical theories (from a proof-theoretical or model-theoretical point of view). It is also true that at least in some cases pure logics are developed to find an answer to a philosophical interpretation. For example, pure modal logics have been motivated by our interest in explaining what is metaphysically necessary or possible. Nevertheless, we would like to argue that there are no intrinsic connections between pure logics and one standard philosophical interpretation, even when one is considering these systems applied to human reasoning.

The opposite point of view has various representatives. For example, David Lewis seems to be someone who holds a canonical philosophical interpretation thesis for pure modal logics. According to him, modal logics talk about real possible worlds (see [14]). We oppose this approach. We would like to draw attention to the fact that one can use Kripkemodels to represent epistemic states of informational scenarios. Or one may be interested in what is obligatory or permitted and use Kripke-models to talk about acceptable worlds. But, similar structures can be used to give the models for intuitionistic logic. Here one can understand these structures as talking about constructive proofs. So, at this point, there is no possibility of adding sufficient restrictions to the theoretical model apparatus that are capable of fixing a single intended philosophical interpretation.

Let us emphasize the point with another example: it is well known that truth values can be interpreted in different ways in the Weak Kleene WK3 matrix: the intermediate value can be interpreted as being meaningless (Bochvar's interpretation) or as being off-topic (Beall's interpretation). Moreover, several works about how to analyze paraconsistent Weak Kleene have recently been published. All of them are examples that involve the systems and show how these features could determine one or more interpretations.

Australasian Journal of Logic (15:2) 2018 Article no. 3.1 
Nevertheless, some philosophers and logicians have adopted the opposite point of view. They have supported the idea that there are ontological or epistemological consequences arising from adopting some pure systems. Another example seems to be Timothy Williamson. He has supported Classical Logic because of this system talks (canonically) about absolute generality. He thinks of a logical theory as a theory of unrestricted generalizations. These generalizations are not specifically about properties of arguments, sentences, propositions; they are generalizations about absolutely all things in the world ([33]). Michael Dummett has adopted a similar approach. From his point of view, classical logic necessarily has ontological commitments; it is committed to metaphysical realism. The use of classical logic in a realm of discourse commits one to realism concerning that discourse. Pure paraconsistent logics are not an exception to this point. For example, analyzing the pure dialectical paraconsistent logic DM, Routley and Meyer affirm:

Whereas intuitionism rejects the negation principle $\neg \neg A \rightarrow A$ dialectical logic DM retains (this principle) but rejects, what intuitionism keeps, $A \rightarrow$ $\neg \neg A$. These formal differences derive from deeper semantical differences, in that whereas intuitionists regard the real world 0 as incomplete (as concerns non-finitary matters) dialecticians take 0 to be inconsistent (beyond local stable regions). ([30, p. 10-11])

This view seems to assume that the logics DM and intuitionistic logic are directly connected with different views about the real world. The idea is that adopting one of the two systems leads directly to two opposing conceptions about the world. We want to reject this thesis.

Of course, there are different philosophical ways of interpreting pure paraconsistent logics. Maybe our evidence is inconsistent and one is interested in reasoning under these conditions. Perhaps one has confirmatory and refutatory evidence for a hypothesis. But, of course, dialetheism is another philosophical interpretation for pure paraconsistent logics. And we are not rejecting the possibility of giving a philosophical interpretation of some paraconsistent logic that adopts dialetheia. This seems to be Priest's point of view and we are not criticizing this approach ([22, p. 67]):

So far I have argued that the mere failure of truth of a sentence is sufficient ground for the truth of its negation; that is, that truth and falsity are mutually exhaustive. The next question is whether they are mutually exclusive. This is obviously the question of whether dialetheism is true, which I have already answered positively in the first part of the book: some sentences are both true and false.

What we are going to reject is that there is a single canonical philosophical interpretation for some pure paraconsistent logic even when one is interested in the standard application of pure logics: the reasoning in natural languages.

So, once the distinction between pure, applied logics and philosophical interpretations were made, we can go back to the definition of paraconsistency. We want to stress the following: paraconsistency is a formal property of some formal system. In the same way as consistency and inconsistency are usually ascribed to formal systems, i.e. it is possible Australasian Journal of Logic (15:2) 2018 Article no. 3.1 
to formally define Post-consistency and negation-consistency, we find that paraconsistency must be defined using some formal method. So as we've seen in the previous section, Urbas [32], among others, tries to define paraconsistency as being capable of supporting inconsistent information. However, this is not what we should look at when seeking a formal definition for the pure logic, but when we are looking for an application for some pure logic; in other words, defining paraconsistency as the possibility of supporting inconsistent theories, as Urbas seems to do, confuses pure logic with applied logic. Because of this, we will stick to what we claimed in the last paragraph of the last section, and we will take the failure of explosion as the distinctive note of paraconsistency. Again, this is not the same as supporting inconsistent theories; the first one is a characteristic of the pure logic, the second one is an application of the logic.

In the next section, we will show that not only have pure and applied logics been confused, but also pure/applied logic with philosophical interpretations. So, dialetheism is one possible philosophical interpretation of some pure logics, as $\mathbf{L P}$.

\section{From paraconsistency to Dialetheism}

As mentioned before, many theorists have thought that the formal notion of paraconsistency requires or calls for the semantical/ontological, depending on the interpretation, notion of dialetheism. The main proposal of this section is to argue against this thesis. So, we support the idea that pure paraconsistent logics do not necessarily imply a dialetheic interpretation. There is no intrinsic feature, e.g. in $\mathbf{L P}$ as pure logic, that allows for the establishing of a dialetheic interpretation as its canonical interpretation. In the following, we will use $\mathbf{L P}$ as the paradigmatic paraconsistent logic, but the idea can be generalized to any paraconsistent logic. So, let us begin by introducing the propositional fragment of the logic LP:

Definition 5.1. The Logic of Paradox LP is the three-valued logic induced by the matrix $\left\langle\mathcal{V}_{\mathbf{L P}}, \mathcal{D}_{\mathbf{L P}}, \mathcal{O}_{\mathbf{L P}}\right\rangle$, where the set of semantic values is $\mathcal{V}_{\mathbf{L P}}=\{\mathbf{t}, \mathbf{i}, \mathbf{f}\}$, the set of designated ones is $\mathcal{D}_{\mathbf{L P}}=\{\mathbf{t}, \mathbf{i}\}$ and $\mathcal{O}_{\mathbf{L P}}=\left\{f_{\mathbf{L P}}^{\neg}, f_{\mathbf{L P}}^{\wedge}, f_{\mathbf{L P}}^{\vee}\right\}$ where these truth-functions are as follows:

\begin{tabular}{|c|c|c|c|c|c|c|c|c|c|}
\hline & $f_{\mathbf{L P}}^{\neg}$ & $f_{\mathbf{L P}}^{\wedge}$ & $\mathbf{t}$ & $\mathbf{i}$ & f & $f_{\mathbf{L} \mathbf{P}}^{\vee}$ & $\mathbf{t}$ & $\mathbf{i}$ & f \\
\hline $\mathbf{t}$ & $f$ & $\mathbf{t}$ & $\mathbf{t}$ & $\mathbf{i}$ & $\mathbf{f}$ & $\mathrm{t}$ & $\mathbf{t}$ & $\mathbf{t}$ & $\mathbf{t}$ \\
\hline $\mathbf{i}$ & $\mathrm{i}$ & $\mathrm{i}$ & $\mathrm{i}$ & $\mathbf{i}$ & $\mathrm{f}$ & $\mathbf{i}$ & $\mathbf{t}$ & $\mathrm{i}$ & $\mathrm{i}$ \\
\hline f & $\mathbf{t}$ & $f$ & f & f & $\mathbf{f}$ & $f$ & $\mathbf{t}$ & $\mathbf{i}$ & f \\
\hline
\end{tabular}

other derived connectives, such as $\rightarrow$, are defined as usual.

So, this is a three-valued presentation of LP. However, so far, this is a pure logic. If we identify a logic with the set of valid inferences, this pure logic can be presented in different ways. In particular, LP can be introduced by relation semantics, tableaux, or sequent-calculus, among others. Of course, by definition, in all of them, the same validities hold. This means that same results can be obtained independently of the special features of the way logics are presented. So, It cannot be argued that what makes a logic like $\mathbf{L P}$ intrinsically dialetheic is the philosophical interpretation of the third value in a three-valued presentation. There is no intrinsically dialetheic value for $\mathbf{L P}$. This is so because there are two-valued LP-models, i.e. two-valued presentations of $\mathbf{L P}$ (using relational semantics).

Australasian Journal of Logic (15:2) 2018 Article no. 3.1 
Secondly, even if we focus on the trivalent presentation, the intermediate value might be interpreted in a non-dialetheic fashion. In other words, to admit three-valued semantics does not compel us to interpret the intermediate semantic value, this pure value, in any particular way.

Of course, one could reply to our position by noting that, although it is correct regarding the relationship between pure logic and its links to philosophical interpretations, the situation is completely different when it fixes the context of an application.

In particular, when we deal with paradoxical sentences, such as the liar sentence:

The sentence $\lambda$ is false

three-valued presentations of $\mathbf{L P}$ allow to assigning the intermediate value to $\lambda$ and its negation, in every model. Thus, one could believe that this sentence (and, of course, its negation) is a glut: true and false in every model, a dialetheia.

Certainly, we are not arguing against dialetheic interpretations of $\mathbf{L P}$. Indeed, we are not rejecting that the idea the pure logic $\mathbf{L P}$ applies to the reasoning that involves the truth predicate of the natural languages and can be philosophically interpreted by dialetheia. Our point is that although perfectly possible, it is not necessary. There are other ways to philosophically interpret the third value of the pure system $\mathbf{L P}$ even when one applies this system to semantic paradoxes. For example, it is perfectly possible to avoid any commitments with dialetheia by adopting an interpretation that involves two forms of assertion: strict and tolerant. According to this interpretation, strictly, the liar and other paradoxical sentences cannot be asserted; but, tolerantly, they can. The same goes for their negations.

Moreover, stressing this point, in [5], Beall and Ripley have offered an analetheic (truthvalue gaps) interpretation for $\mathbf{L P}$. In fact, they take $\mathbf{L P}$ and show how to accommodate a truth predicate. From the analetheic point of view, paradoxical sentences lack truthvalue. So, what the authors claim is that it is perfectly possible to give a parallel nondialetheic version of $\mathbf{L P}$ that enjoys similar positive properties, when dealing with semantic vocabulary.

Our points should be taken into account to evaluate some current discussions on the connections between paraconsistency and dialetheism. For example, recently, Beziau in [8] has argued that $\mathbf{L P}$ commits the dialetheist to trivialism. According to him, firstly "any atomic formula $\mathrm{S}$ [in $\mathbf{L P}]$ is a dialetheia" ([8, p. 52]) and, secondly, "every molecular formula is a dialetheia" in LP ([8, p. 54]). Nevertheless, Martin in [15, p.11] has replied that:

"all Beziau has shown is that every formula in $\mathbf{L P}$ is a dialetheia in an interpretation, which in principle is no different to showing that the proposition 'Barack Obama is a fried egg' is true in some interpretations in classical logic. Neither entail truth simpliciter. To show that LP commits the dialetheist to all formulae being dialetheia would require showing that in every interpretation in which some formula is a dialetheia, every formula is a dialetheia. Yet, this is trivially not true of $\mathbf{L P}$, for it is a paraconsistent logic"

Australasian Journal of Logic (15:2) 2018 Article no. 3.1 
Obviously, Martin is right about the trivialism point. But both are wrongly supporting the thesis that we are rejecting: there is an only one canonical interpretation for LP. Our point is that there is nothing intrinsic to LP that connects this pure logic with a single philosophical interpretation (dialetheism).

Another reason that we would like to give against the one logic, one canonical philosophical interpretation is the following: $\mathbf{L P}$ and all other subclassical logics allow for the recapturing of classical logic through operators and other mechanisms (Barrio [1] , JC Beall [4, pp. 755-764] and Omori [17]). As Omori claims [17, p.117]: "LP is not dialetheic since it is a subsystem of classical logic".

We should be impressed by translations between logics. This is a general phenomenon. And it is a remarkable result that $\mathbf{C L}$ can be expressed inside some non-classical logics: its rules and laws can be captured as acceptable under certain conditions. This is the case with LP. Most paraconsistent logicians do not propose a wholesale rejection of CL. They usually accept the validity of classical inferences in consistent contexts. CL is recovered inside this system only taking into consideration only classical values and classical definition of validity as truth preservation. In this way, one can capture $\mathbf{C L}$ inside $\mathbf{L P}$ for some contexts. Besides, classical negation is definable in $\mathbf{L P}$ adding a consistency operator $\circ^{7}$. Recovering classical negation inside LP seems to allow different philosophical interpretations for the same pure system. Therefore, it seems that it is necessary to say that $\mathbf{L P}$ cannot be really interpreted as capturing the idea that there are true contradictions.

Finally, one could be interested in adding some conditions to $\mathbf{L P}$ to avoid this last result. Omori in [17] presents a new paraconsistent logic by expanding $\mathbf{L P}$. The new logic, referred to as $\mathbf{d L P}$ (dialetheic $\mathbf{L P}$ ), is obtained by combining connectives from different views of paraconsistency (connexive logics and LFIs). This pure logic has some interesting distinctive features. As Omori shows the propositional fragment of $\mathbf{d L P}$ is Post complete. Let us present the propositional fragment of this logic.

Definition 5.2. dLP is the three-valued logic induced by the matrix $\left\langle\mathcal{V}_{\mathbf{d L P}}\right.$, $\left.\mathcal{D}_{\mathbf{d L P}}, \mathcal{O}_{\mathrm{dLP}}\right\rangle$, where the set of semantic values is $\mathcal{V}_{\mathbf{d L P}}=\{\mathbf{t}, \mathbf{i}, \mathbf{f}\}$, the set of designated ones is $\mathcal{D}_{\mathbf{d L P}}=\{\mathbf{t}, \mathbf{i}\}$ and $\mathcal{O}_{\mathbf{d L P}}=\left\{f_{\mathbf{d L P}}, f_{\mathbf{d L P}}^{\wedge}, f_{\mathbf{d L P}}^{\vee}, f_{\mathbf{d L P}}, f_{\mathbf{d L P}}^{\circ}\right\}$ where these truth-functions are as follows:

\begin{tabular}{|c|c|c|c|c|c|c|c|c|c|c|c|c|c|}
\hline & $f_{\mathrm{dLP}}^{\neg}$ & $f_{\mathrm{dLP}}^{\circ}$ & $f_{\mathrm{dLP}}^{\wedge}$ & $\mathbf{t}$ & i & f & $f_{\mathrm{dLP}}^{\vee}$ & $\mathbf{t}$ & i & $\mathbf{f}$ & $f_{\mathrm{dL} P}$ & $\mathbf{t}$ & i \\
\hline $\mathbf{t}$ & f & $\mathbf{t}$ & $\mathbf{t}$ & $\mathbf{t}$ & $\mathbf{i}$ & f & $\mathrm{t}$ & $\mathbf{t}$ & $\mathrm{t}$ & $\mathbf{t}$ & $\mathrm{t}$ & $\mathbf{t}$ & i \\
\hline $\mathbf{i}$ & i & f & $\mathbf{i}$ & $\mathbf{i}$ & $\mathbf{i}$ & f & $\mathbf{i}$ & $\mathbf{t}$ & $\mathbf{i}$ & $\mathbf{i}$ & $\mathbf{i}$ & $\mathbf{t}$ & $\mathbf{i}$ \\
\hline$f$ & $\mathbf{t}$ & $\mathbf{t}$ & f & f & f & f & $\mathbf{f}$ & $\mathbf{t}$ & i & f & f & i & $\mathbf{i}$ \\
\hline
\end{tabular}

It is worth noting that $\circ$ is a recovery or consistency operator, which means that $\mathbf{d L P}$ is an LFI. Also, the other distinctive feature, compared with $\mathbf{L P}$ is that implication is

\footnotetext{
${ }^{7}$ Roughly speaking, using common self-referential mechanisms, this result is possible only if the language does not have its own truth predicate. But as Barrio, Pailos and Szmuc point out in [2], this result could change by adopting Goodship's project. In this case, it is possible to add a consistency operator to LP and define a classical negation in a language with transparent truth.
}

Australasian Journal of Logic (15:2) 2018 Article no. 3.1 
expressed via a primitive connective. It is clear that $\mathbf{d L P}$ is not subclassical. Hence, unlike $\mathbf{L P}$, $\mathbf{d L P}$ could be a good candidate to be dialetheic.

Nevertheless, even though $\mathbf{d L P}$ is not subclassical, we want to emphasize at this point that this result is not sufficient to have only one canonical philosophical interpretation. Even the logical resources are compatible with dialetheias, the pure system is also compatible with an epistemic interpretation concerning contradictory evidence. That is, not even $\mathbf{d L P}$ can capture only one dialetheic interpretation.

\section{From dialetheism to Paraconsistency}

In Section 2 we presented the definition of dialetheism, and, in a nutshell, it was defined as accepting true contradictions (either from an ontological point of view or a semantical one, or both). On the other hand, in Section 3 we summarized many possible definitions of paraconsistency. The moral of that discussion is that, whatever a parconsistent logic may be, a logic is paraconsistent only if it fails to validate the rule of explosion, i.e. $A, \neg A \not \models B$, for some formulae $A, B$. In other words, if a logic validates explosion it is not paraconsistent at all. Also, negation plays a crucial role in this formulation of paraconsistency; it seems impossible for a logic to be paraconsistent and to lack some negation. So far, in the literature, dialetheism has usually been related with paraconsistency. Thus, many philosophers have considered it impossible to be dialetheist and not to accept a paraconsistent logic. Just for giving a recent example, Omori claims in [17, p. 112]:

As is well known, paraconsistent logics are independent of dialetheism in general. More specifically, dialetheism calls for paraconsistent logic, but not the other way around.

So, as we argued earlier, we agree with Omori when he claims that paraconsistent logic does not require a dialetheic interpretation.

However, in this section, we will show that it is possible to embrace contradictions in a non-paraconsistent frame. In order to do so, we will give some examples of this thesis. In particular, with the development of substructural logics, we find examples of logics that can possibly accept contradictions, and at the same time accepting the principle of explosion. One of these logics is ST, developed in [25],[10], [26]. Let us first introduce a sequent calculus for the logic $\mathbf{S T}$.

Definition 6.1. (ST) Let $\Gamma, \Delta, \Pi$, and $\Sigma$ be (finite) sets of formulas, let $\phi$ and $\psi$ be formulas. The system $\mathbf{S T}$ is given by the following rules:

\section{Structural rules}

$$
\text { Reflexivity } \overline{\phi \Rightarrow \phi}
$$

Australasian Journal of Logic (15:2) 2018 Article no. 3.1 


$$
\mathrm{KL} \frac{\Gamma \Rightarrow \Delta}{\Gamma, \Sigma \Rightarrow \Delta} \quad \mathrm{KR} \frac{\Gamma \Rightarrow \Delta}{\Gamma \Rightarrow \Pi, \Delta}
$$

\section{Operational rules}

$\mathrm{L} \wedge \frac{\Gamma, \phi, \psi \Rightarrow \Delta}{\Gamma, \phi \wedge \psi \Rightarrow \Delta}$

$\mathrm{L} \vee \frac{\Gamma, \phi \Rightarrow \Delta \quad \Sigma, \psi \Rightarrow \Pi}{\Gamma, \Sigma \phi \vee \psi \Rightarrow \Pi, \Delta}$

$\mathrm{L} \neg \frac{\Gamma, \Rightarrow \phi, \Delta}{\neg \phi, \Gamma \Rightarrow \Delta}$

$$
\begin{aligned}
& \mathrm{R} \wedge \frac{\Gamma \Rightarrow \Delta, \phi \quad \Sigma \Rightarrow \Pi, \psi}{\Gamma, \Sigma \Rightarrow \Delta, \Pi, \phi \wedge \psi} \\
& \mathrm{R} \vee \frac{\Gamma, \phi, \psi \Rightarrow \Delta}{\Gamma, \phi \vee \psi \Rightarrow \Delta} \\
& \mathrm{R} \neg \frac{\Gamma, \phi \Rightarrow \Delta}{\Gamma, \Rightarrow \neg \phi, \Delta}
\end{aligned}
$$

Using this logic, Ripley [25] has shown that it's possible to build a transparent theory of truth, avoiding triviality ${ }^{8}$. So, let us introduce this theory:

Definition 6.2. $(S T T T)$ Let $\Gamma$ and $\Delta$ be (finite) sets of formulas, let $\phi$ be a formula, and let $\operatorname{Tr}$ be the truth predicate. The system STTT is $\mathbf{S T}$ plus the following rules:

$$
\mathrm{L} \operatorname{Tr} \frac{\Gamma, \phi \Rightarrow \Delta}{\operatorname{Tr}(\ulcorner\phi\urcorner), \Gamma \Rightarrow \Delta} \quad \operatorname{RTr} \frac{\Gamma \Rightarrow \phi, \Delta}{\Gamma \Rightarrow \operatorname{Tr}(\ulcorner\phi\urcorner), \Delta}
$$

In this theory, we can express the liar sentence. So we will take the sentence $\lambda$ to be equivalent to $\neg \operatorname{Tr}(\ulcorner\lambda\urcorner)$, i.e. $\lambda$ says about itself that it's not true. With this sentence in play, we can prove $\Rightarrow \operatorname{Tr}(\ulcorner\lambda\urcorner)$ and $\Rightarrow \neg \operatorname{Tr}(\ulcorner\lambda\urcorner)$, as follows:

$$
\begin{array}{r}
\mathrm{Id} \frac{\operatorname{Tr}(\ulcorner\lambda\urcorner) \Rightarrow \operatorname{Tr}(\ulcorner\lambda\urcorner)}{\mathrm{R} \neg \frac{\operatorname{Tr}(\ulcorner\lambda\urcorner), \neg \operatorname{Tr}(\ulcorner\lambda\urcorner)}{\Rightarrow \operatorname{Tr}(\ulcorner\lambda\urcorner)}} \\
\mathrm{R} \operatorname{Tr} \frac{\Rightarrow}{\Rightarrow \ln }
\end{array}
$$

and

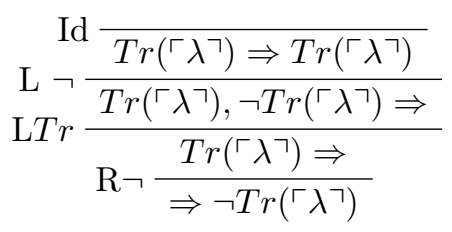

\footnotetext{
${ }^{8}$ We assume some way of achieving self-reference. For instance, we can get a stock of distinguished constants that are interpreted in the same way in every model and work as names of the sentences. Notationally, we will take $\ulcorner\phi\urcorner$ to be the name of the sentence $\phi$. It's not possible to give all the details here, so we recommend the reader to see, for instance, [25].
}

Australasian Journal of Logic (15:2) 2018 Article no. 3.1 
Therefore, in this theory we can explicitly represent a dialetheia, i.e. we prove that a sentence is true and false (since we can identify its falsity with the negation of its truth). Moreover, this theory seems to be a good candidate for those who support some kind of semantical dialetheism, because the truth predicate allows for expressing the main thesis of this position. It is worth pointing out that the way of avoiding triviality from this is the rejection of the rule of Cut:

$$
\operatorname{Cut} \frac{\Gamma \Rightarrow \phi, \Delta \quad \Pi, \phi \Rightarrow \Sigma}{\Gamma, \Pi \Rightarrow \Delta, \Sigma}
$$

So, Cut is not admissible on pain of triviality. On the other hand, since the base logic of $S T T T$ is $S T$, the theory is explosive:

$$
\begin{gathered}
\mathrm{Id} \\
\mathrm{R} K \frac{\overline{\phi \Rightarrow \phi}}{\phi, \neg \phi \Rightarrow}
\end{gathered}
$$

for any pair of formulae of the language $\phi$ and $\psi$.

Therefore, we have a dialetheic theory that is not paraconsistent. Not only that, but with STTT it's possible to be committed to the so-called "semantical dialetheism", and not necessarily to ontological dialetheism.

However, although we have presented a theory adding a truth predicate over a nonparaconsistent logic that allows a dialetheist interpretation, some people could find the way of doing it to be suspicious, especially since we are not working with a logic strictu sensu but with a theory. So, in what follows, we also will show that a logic can be compatible with a dialetheist intepretation and be non-paraconsistent.

The logic we will work with is an extension of $\mathbf{S T}$ with a constant $\lambda^{9}$ :

Definition 6.3. $\left(S T_{\lambda}\right)$ Let $\Gamma$ and $\Delta$ be (finite) sets of formulas, let $\phi$ be a formula. The system $S T_{\lambda}$ is $\mathbf{S T}$ plus the following rules:

$$
\mathrm{L} \lambda \frac{\Gamma, \Rightarrow \lambda, \Delta}{\lambda, \Gamma \Rightarrow \Delta} \quad \mathrm{R} \lambda \frac{\Gamma, \lambda \Rightarrow \Delta}{\Gamma, \Rightarrow \lambda, \Delta}
$$

In $S T_{\lambda}$, we can prove a sentence and its negation, $\Rightarrow \lambda$ and $\Rightarrow \neg \lambda$, in the following way:

$$
\begin{array}{r}
\mathrm{Id} \\
\mathrm{L} \lambda \frac{\lambda \Rightarrow \lambda}{\lambda \neg \frac{\lambda \Rightarrow}{\Rightarrow \neg \lambda}}
\end{array}
$$

and

\footnotetext{
${ }^{9}$ For this constant, we use the same letter as the liar sentence, since the intended interpretation of this constant is a contradictory object, such as the liar sentence.
}

Australasian Journal of Logic (15:2) 2018 Article no. 3.1 


$$
\operatorname{Id} \frac{}{\frac{\lambda \Rightarrow \lambda}{\Rightarrow \lambda}}
$$

Of course, $S T_{\lambda}$ is not trivial, since explicitly rejects the rule of Cut:

$$
\operatorname{Cut} \frac{\Gamma \Rightarrow \phi, \Delta \quad \Pi, \phi \Rightarrow \Sigma}{\Gamma, \Pi \Rightarrow \Delta, \Sigma}
$$

So, the logic is perfectly compatible with a dialetheist position. However, the logic is not paraconsistent since it's an extension of classical logic:

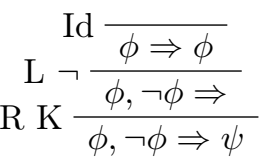

In this way, it seems that dialetheism doesn't call for paraconsistency.

As we have seen in Section 3, every definition of paraconsistency shares the rejection of explosion. And, $S T T T$ and $S T_{\lambda}$ validate the rule of explosion. So, both logics are clearly non-paraconsistent.

Finally, let's mention that recently, Barrio, Pailos and Szmuc ([3]) have proposed a generalization of the notion of paraconsistency. They analyze the meta-inferential formulation of Explosion (Meta-Explosion):

$$
\text { Meta-Explosion } \frac{\Rightarrow A \quad \Rightarrow \neg A}{\Rightarrow B}
$$

Therefore, the idea is to define paraconsistency in terms of failure of Explosion or MetaExplosion. According to this view, it is possible to have logics which have a non-uniform policy towards paraconsistency. For instance, $S T_{\lambda}$ would be paraconsistent at the metainferential level, but non-paraconsistent at the inferential level. In any case, again, usual definitions of paraconsistency focus on the inferential level (the level of the logic, so to say) and the inferential version of Explosion is valid in $S T_{\lambda}{ }^{10}$.

\footnotetext{
${ }^{10}$ It is not our aim to close the discussion of this section with a categorical assertion on how to define paraconsistency, but rather to conditionalize our claims to the usual and at certain extent accepted minimal criteria used to determine whether a logic is or not paraconsistent, i.e. failure of explosion (see Section 3 for details). Under this criterion none of the logics presented above is paraconsistent.
}

Australasian Journal of Logic (15:2) 2018 Article no. 3.1 


\section{Conclusion}

In this paper, we have argued against the existence of any essential relationship between pure/applied paraconsistent logics and their philosophical interpretations. In particular, we tried to show that paraconsistency and dialetheism are not so intrinsically connected as usually assumed. In order to do so, we firstly reviewed the most important attempts to define dialetheism and paraconsistency. This led us to one of the problems of our task: there is no consensus about how to define paraconsistency. However, what we found that common to almost every proposal is the rejection of the inferential rule of explosion.

Next, we argued against the idea that there is a single canonical philosophical interpretation for some pure logic, focusing on the dialetheist interpretation of paraconsistent logics. In this sense, in order to strengthen the point, using $\mathbf{L P}$ as the paradigmatic example of a paraconsistent logic, we have shown that it is perfectly possible to adopt a paraconsistent logic and reject dialetheism. Moreover, we presented two examples in order to justify the other direction of the implication: it's possible to be a dialetheist and to adopt a non-paraconsistent logic.

As a concluding remark, we want to emphasize that, contrary to what a first view of this paper might suggest, it was not our aim to criticize dialetheism in general or some philosophical interpretation of some particular paraconsistent logic. In fact, we consider dialetheism to be a plausible philosophical interpretation of LP. Our point was just to argue that this relation is not as close as many theorists have claimed.

\section{ACKNOWLEDGMENTS}

We would like to thank the members of the Buenos Aires Logic Group for helpful feedback concerning previous versions of this paper. Our thanks also go to Hitoshi Omori and one anonymous reviewer for their helpful suggestions and comments on a previous version of this article. Finally, we would also like to thank the editors of the special issue on the work of Richard Sylvan (Routley), especially to Filippo Casati and Graham Priest, for their support through the editorial process.

\section{REFERENCES}

[1] Eduardo Barrio. Models and Proofs: LFIs without a Canonical Interpretation. Principia, Forthcoming.

[2] Eduardo Alejandro Barrio, Federico Matias Pailos, and Damian Enrique Szmuc. A paraconsistent route to semantic closure. Logic Journal of the IGPL, 25(4):387-407, 2017.

[3] Edwardo Barrio, Frederico Pailos, and Damián Szmuc. What is a paraconsistent logic. In Jacek Malinowski and Walter Carnielli, editors, Between Consistency and Inconsistency. Trends in Logic: Studia Logica series, Forthcoming.

[4] Jc Beall. A simple approach towards recapturing consistent theories in paraconsistent settings. The Review of Symbolic Logic, 6(4):755-764, 2013.

[5] Jc Beall and David Ripley. Analetheism and dialetheism. Analysis, 64(281):30-35, 2004.

Australasian Journal of Logic (15:2) 2018 Article no. 3.1 
[6] Jeffrey C Beall. True and false - as if. In Graham Priest, Jeffrey C Beall, and Bradley P Armour-Garb, editors, The law of non-contradiction: New philosophical essays, pages 197-216. Oxford University Press, 2004.

[7] Francesco Berto. Is dialetheism an idealism? The russellian fallacy and the dialetheist's dilemma. dialectica, 61(2):235-263, 2007.

[8] Jean-Yves Beziau. Trivial dialetheism and the logic of paradox. Logic and Logical Philosophy, 25(1):51-56, 2015.

[9] Ottavio Bueno. Can a paraconsistent theorist be a logical monist? In M. E. Coniglio W. A. Carnielli and I. M. L. D'Ottaviano (eds.), editors, Paraconsistency: The Logical Way to the Inconsistent, pages 535-552. Marcel Dekker, 2002.

[10] Pablo Cobreros, Paul Égré, David Ripley, and Robert Van Rooij. Reaching transparent truth. Mind, 122(488):841-866, 2013.

[11] Newton CA Da Costa et al. On the theory of inconsistent formal systems. Notre dame journal of formal logic, 15(4):497-510, 1974.

[12] Michael De and Hitoshi Omori. Classical negation and expansions of belnap-dunn logic. Studia Logica, 103(4):825-851, 2015.

[13] I Johansson. The minimal calculus, a reduced intuitionistic formalism. Compositio Mathematica, 4:119-136, 1936.

[14] David Lewis. On the plurality of worlds, volume 322. Oxford, 1986.

[15] Ben Martin. In defence of dialetheism: A reply to Beziau and Tkaczyk. Logic and Logical Philosophy, 2017.

[16] Carlos Oller. Paraconsistent logic. Notae Philosophicae Scientiae Formalis, 3(1):1-7, 2014.

[17] Hitoshi Omori. From paraconsistent logic to dialetheic logic. In Logical Studies of Paraconsistent Reasoning in Science and Mathematics, pages 111-134. Springer, 2016.

[18] G Priest and J Woods. Paraconsistency and dialetheism. the many valued and nonmonotonic turn in logic, 2007.

[19] Graham Priest. The logic of paradox. Journal of Philosophical logic, 8(1):219-241, 1979.

[20] Graham Priest. Logic: one or many? Logical consequence: Rival approaches, pages 23-38, 2001.

[21] Graham Priest. Doubt Truth to be a Liar. Oxford University Press, 2006.

[22] Graham Priest. In Contradiction: A Study of the Transconsistent. Oxford University Press, 2006.

[23] Graham Priest and Francesco Berto. Dialetheism. In Edward N. Zalta, editor, The Stanford Encyclopedia of Philosophy. Metaphysics Research Lab, Stanford University, Spring 2017 edition, 2017.

[24] Greg Restall. Paraconsistent logics! Bulletin of the Section of Logic, 26(3):156-163, 1997.

[25] D. Ripley. Conservatively Extending Classical Logic with Transparent Truth. Review of Symbolic Logic, 5(2):354-378, 2012.

[26] David Ripley. Paradoxes and failures of cut. Australasian Journal of Philosophy, 91(1):139-164, 2013.

Australasian Journal of Logic (15:2) 2018 Article no. 3.1 
[27] David Ripley. Paraconsistent logic. Journal of Philosophical Logic, 44(6):771-780, 2015.

[28] Richard Routley. Dialectical logic, semantics and metamathematics. Erkenntnis, 14(3):301-331, 1979.

[29] Richard Routley. Exploring Meinong's jungle and beyond: an investigation of noneism and the theory of items. Departmental Monograph, 1980.

[30] Richard Routley and Robert K Meyer. Dialectical logic, classical logic, and the consistency of the world. Studies in East European Thought, 16(1):1-25, 1976.

[31] Barry Hartley Slater. Paraconsistent logics? Journal of Philosophical logic, 24(4):451454, 1995.

[32] Igor Urbas. Paraconsistency. Studies in Soviet Thought, 39(3-4):343-354, 1990.

[33] Timothy Williamson. Semantic paradoxes and abductive methodology. In Bradley Armour-Garb, editor, Reflections on the Liar, pages 325-346. Oxford University Press, 2017.

IIF-SADAF-CONICET - University of Buenos Aires

Current address: Bulnes 642, Buenos Aires, Argentina

E-mail address: eabarrio@gmail.com

IIF-SADAF-CONICET - University of Buenos Aires

Current address: Bulnes 642, Buenos Aires, Argentina

E-mail address: brunohoraciodare@gmail.com

Australasian Journal of Logic (15:2) 2018 Article no. 3.1 